RESEARCH ARTICLE

\title{
Evaluation of Rifampicin-resistant Tuberculosis in Pediatric Patients by GeneXpert MTB/RIF
}

\author{
Ashish William, Yogita Rai, Ravinder Kaur \\ Department of Microbiology, Lady Hardinge Medical College \& Associated Hospitals, New Delhi, India
}

\begin{abstract}
Objectives: India has the largest burden of MDR-TB worldwide, with an annual incidence of 1,30000 patients. Multidrug-resistant tuberculosis (MDR-TB) carries a poor prognosis, a high mortality rate, and treatment success rates as low as $65 \%$. The mortality in India is estimated to be about 480,000 per year. The aim of the study was to evaluate Rifampicin-resistant Tuberculosis and its distribution by GeneXpert in a tertiary care hospital.
\end{abstract}

Methods: A total of 2864 samples were processed from the patients attending outpatient departments and indoor wards as per the pediatrician's request. Acid-fast bacilli (AFB) smear microscopy was done on all samples by Acid-fast staining for early diagnosis followed by GeneXpert MTB/RIF (CBNAAT) testing. Specimens were transported and stored at $2-8{ }^{\circ} \mathrm{C}$ prior to processing for CBNAAT. Results were read and reported within 2 hours.

Results: A total of 2864 samples were tested for TB using CBNAAT (including 645 [22.5\%] extra-pulmonary and 2219 [77.5\%] pulmonary samples). The test results were positive in a total of 346 (12\%) samples by CBNAAT. The positivity is highest in the age group $>10$ years and in sputum samples $(37.28 \%)$ followed by pus samples $(23.26 \%)$ in pulmonary and extrapulmonary distribution. Out ZN staining was positive in 244 (8.5\%) specimens. There were 102 (29.5\%) CBNAAT positive specimens which showed negative results for Acid-fast bacilli (70.52\% Sensitivity). Among 346 TB-positive patients, $10.46 \%$ samples were pulmonary and $17.67 \%$ were extrapulmonary. It was observed that in positive CBNAAT patients, the prevalence of Rifampicin resistance was $12.72 \%$ i.e. 44 samples which include $31(13.36 \%)$ and $13(11.40 \%)$ pulmonary and extra-pulmonary samples respectively. Among the Rifampicin resistant samples, there were $29.55 \%$ samples that were extrapulmonary.

Conclusion: GeneXpert MTB/RIF is a very rapid diagnostic assay that provides information regarding the mutation pattern of RIF resistance in MTB isolates. J Microbiol Infect Dis 2021; 11(2):81-87.

Keywords: CBNAAT, Tuberculosis, GeneXpert MTB/RIF

\section{INTRODUCTION}

Tuberculosis is considered one of the major causes of mortality worldwide [1]. India accounts for the highest-burden of TB (27\%) and MDR-TB $(24 \%)$ as per the Global Tuberculosis Report 2018 [2]. Drug-resistant tuberculosis is caused by genetic mutation of bacilli, an inadequate or poorly administered treatment regimen, and weak services program that leads to delay in detection and effective treatment of drug resistance and are unequipped to support patients to keep adherence to treatment [3].
Multidrug-resistant tuberculosis (MDR-TB), defined as tuberculosis caused by strains of Mycobacterium tuberculosis (MTB) resistant to the two most important antituberculous drugs isoniazid and rifampicin - carries a poor prognosis, a high mortality rate, and treatment success rates as low as $65 \%$ [4]. The mortality in India is estimated to be about 480,000 per year [5]. The exact data of pediatric MDR cases is not available due to the is not There is variation in the viral content of tuberculosis. As it can be a low viral load that limits the detection rate of Multidrug-resistant Tuberculosis. The difficulty in

Correspondence: Dr. Ashish William, Department of Microbiology, Lady Hardinge Medical College \& Associated Hospitals, New Delhi, India 
the diagnosis of the disease can be observed due to the low bacterial content [6]. According to some studies in 2010, there has been an estimation of $850,000-1,000,000$ cases of tuberculosis, out of which cases of MDR-TB had been observed to be around 25,000-32,000 [78].

Extrapulmonary TB accounts for about $25 \%$ of all cases of TB and an even higher percentage of cases in children and in people who are immunocompromised.

Diagnosing extrapulmonary TB is often challenging, requiring the clinician to obtain specimens for microscopy, culture, and histopathology from the suspected sites of involvement [9]. Diagnosis of pulmonary TB in children is also challenging, more so in resource-limited, tuberculosisendemic countries, and is largely based on clinical and radiological findings and medical history [10]. Isolation of mycobacteria by culture, while considered as the gold standard for diagnosing TB, takes 4-8 weeks and often requires expensive and sophisticated laboratory facilities which cannot be afforded in most resource-limited settings [11]. Due to poor sensitivity and specificity, it is not recommended to use antigen and antibody TB ELISA tests for the diagnosis of tuberculosis [12]. The latent TB infection is being detected by Interferon-gamma release assays (IGRAs) which had replaced skin tests in low prevalence countries. However, these tests are expensive and lack in differentiation the TB infection from disease. In case of a high burden, the use of these tests is still not clear [13].

There is quite a variation seen in the specificities and sensitivities of the polymerase chain reaction (PCR) based diagnostic tests [13]. Further, these tests involve multiple manual steps and long turnaround times, making them unsuitable for decentralized deployment. A series of meta-analyses have shown cartridgebased nucleic acid amplification test (CBNAAT)/ Xpert MTB/ RIF to have a high specificity with variable sensitivity in different types of specimens for TB diagnosis [14]. In 2013, the WHO endorsed the use of CBNAAT for TB diagnosis in pediatric presumptive pulmonary and extra-pulmonary tuberculosis (EPTB) cases [15].

The recent introduction of the CBNAAT assay has significantly revolutionized the diagnostics of tuberculosis in adults, but its application for the diagnosis of pediatric TB is under evaluation. To date, there are only a few studies on the application of CBNAAT for the diagnosis of pediatric tuberculosis in India, more so in eastern India. The Gene Xpert MTB/Rif assay is a cartridge-based fully automated NAAT (nucleic acid amplification test) for TB case detection and rifampicin resistance testing, suitable for use in disease endemic countries. This nucleic acid amplification test (CBNAAT) is a recently introduced polymerase chain reaction-based method for the detection of TB. It is an MTBspecific automated, cartridge-based nucleic acid amplification assay, having fully integrated and automated amplification and detection using real-time PCR, providing results within 100 minutes [16]. It has a highly specific primer and five unique molecular probes to target the rpo $B$ gene of $M$. tuberculosis, which is the critical gene associated with rifampicin resistance [17]. No cross-reactions have been observed with many other bacterial species tested, including a comprehensive band of mycobacteria, thereby excluding non-tubercular bacteria. This study was conducted for the knowledge of the pattern of mutations present in RIF-resistant isolates which can provide information regarding the epidemiology of RIF-resistant MTB isolates of this particular area.

The aim of the study was to evaluate Rifampicinresistant Tuberculosis and its distribution by GeneXpert in a tertiary care hospital.

\section{METHODS}

This retrospective study was conducted in the Department of Microbiology, Lady Hardinge Medical College, New Delhi from January 2019 to December 2019. The Lady Hardinge Medical College has accepted samples for CBNAAT testing from connected 377 bedded Kalawati Saran Children Hospital which is limited to pediatric patients. The retrospective data of 1 year was collected from the patients' records maintained in the laboratory. In the study period, a total of 2864 samples were collected and processed from the pediatric patients attending outpatient departments and indoor wards as per pediatrician request in the age group. Acid-fast bacilli (AFB) smear microscopy was done on all samples by Acid-fast staining for early diagnosis followed by CBNAAT testing. 


\section{Specimen Collection and Processing}

Pulmonary samples and Extrapulmonary samples (CSF, Ascitic Fluid, Pus, Pleural Fluid, Urine, Synovial Aspirate, Lymph node aspirates, Pericardial fluid) were collected in leak-proof sterile containers and under aseptic precautions, wherever necessary. The samples were processed as soon as possible. In case of delay, they were refrigerated at $4^{\circ} \mathrm{C}$ for not more than 24 hours and then processed accordingly. All specimen processing was done in Biosafety cabinet class II A2.

\section{AFB Staining}

Ziehl-Neelsen (ZN) staining was performed according to the standard protocol [18]. From each sample, a smear was made on a new, clean, unscratched glass slide. Smear was allowed to air-dried and fixed by heat then the slide was stained by Z-N stain and examined under immersion oil (x100). Acid-fast bacilli appear bright red with a beaded appearance.

\section{GeneXpert}

Processing of specimens for CBNAAT was done as per standard protocol given by WHO [19]. According to the standard operating procedure of GeneXpert MTB/RIF, the sampling reagent (containing $\mathrm{NaOH}$ and isopropanol) was added at 2:1 ratio to the sample and then incubated for 10 minutes at room temperature with intermittent vigorous shaking After that $2 \mathrm{ml}$ of sample reagent mixture was transferred into GeneXpert cartridge and loaded into GeneXpert instrument. Results were obtained after 2 hours, the presence or absence of MTB was reported by the systems with the bacterial load as very low, low, medium, or high and simultaneously giving results for susceptibility to rifampicin drug.

\section{RESULTS}

A total of 2864 samples were tested for TB using CBNAAT (including 645 [22.5\%] extrapulmonary and 2219 [77.5\%] pulmonary samples). The test results were positive in a total of $346(12 \%)$ samples by CBNAAT. The positivity is highest in age group $>10$ years (44.8\%) followed by $6-10$ years $(37.6 \%),<=2$ years $(11.6 \%)$, and $3-5$ years $(6 \%)$ with majority occurrence of Tuberculosis in the females $183(53 \%)$. The specimen distribution in CBNAAT positive is contributed maximum by Sputum samples $(37.9 \%)$ followed by Pus samples (23.7\%), Gastric aspirate (18.2\%), Lymph node aspirates (17.6\%), CSF (2\%), Pleural fluid $(0.9 \%)$, Bronchoalveolar lavage (0.9\%) and Ascitic fluid (0.6\%) (Figure 2). ZN staining was positive in 244 (8.5\%) specimens. Among extrapulmonary samples, maximum positivity is seen in Lymph node aspirates (44.79\%).

Table 1. Age distribution of GeneXpert MTB/RIF positive samples ( $\mathrm{N}=346)$.

\begin{tabular}{lc}
\hline Age Groups & GeneXpert MTB/RIF Positive \\
\hline$\leq 2$ Years & $40(11.6 \%)$ \\
$3-5$ Years & $21(6 \%)$ \\
$6-10$ Years & $130(37.6 \%)$ \\
$>10$ Years & $155(44.8 \%)$
\end{tabular}

There were 102 (29.5\%) CBNAAT positive specimens which showed negative results for Acid-fast bacilli (Table 2). With CBNAAT as the gold standard, the sensitivity and specificity of the $\mathrm{ZN}$ staining were observed to be $70.52 \%$ and $100 \%$ respectively while the positive predictive value and negative predictive value of ZN staining were found to be $100 \%$ and $96.1 \%$ respectively. Among the TB-positive patients, $10.46 \%$ samples were pulmonary and $17.67 \%$ were extrapulmonary (Figure 1). The Rifampicin resistance was seen in 44(12.72\%) patients which included $29.55 \%$ extrapulmonary samples (Figure 3). Among the CBNAAT positive samples, the prevalence of Rifampicin resistance was $31(13.36 \%)$ and $13(11.40 \%)$ in pulmonary and extra-pulmonary respectively (Table 3) with maximum resistance seen in the Sputum samples (50\%) and followed by Pus (34\%), lymph node aspirates (7\%) and Gastric Aspirates (5\%).

Table 2. Distribution of positivity of $\mathrm{ZN}$ staining in comparison to GeneXpert MTB/RIF samples $(\mathrm{n}=2864)$.

\begin{tabular}{lccc}
\hline Variables & $\begin{array}{c}\text { CBNAAT } \\
\text { Positive }\end{array}$ & $\begin{array}{c}\text { CBNAAT } \\
\text { Negative }\end{array}$ & Total \\
\hline $\begin{array}{l}\text { ZN Staining } \\
\text { Positive }\end{array}$ & 244 & 0 & 244 \\
$\begin{array}{l}\text { ZN Staining } \\
\text { Negative }\end{array}$ & 102 & 2518 & 2620 \\
Total & 346 & 2518 & 2864 \\
\hline
\end{tabular}




\section{DISCUSSION}

CBNAAT (Xpert MTB/RIF assay) is a very rapid and accurate diagnostic tool for the early detection of TB. In our study prevalence of Mycobacterium Tuberculosis by CBNAAT among 2864 samples was observed to be 346
$(12 \%)$. This is similar to studies done in Ethiopia (13.6\%) [20] and South Africa (13\%) [21] and Uganda (14\%) [22]. There has been increasing positivity of tuberculosis in the age group of $>10$ years [23] which is similar to the finding in our study.

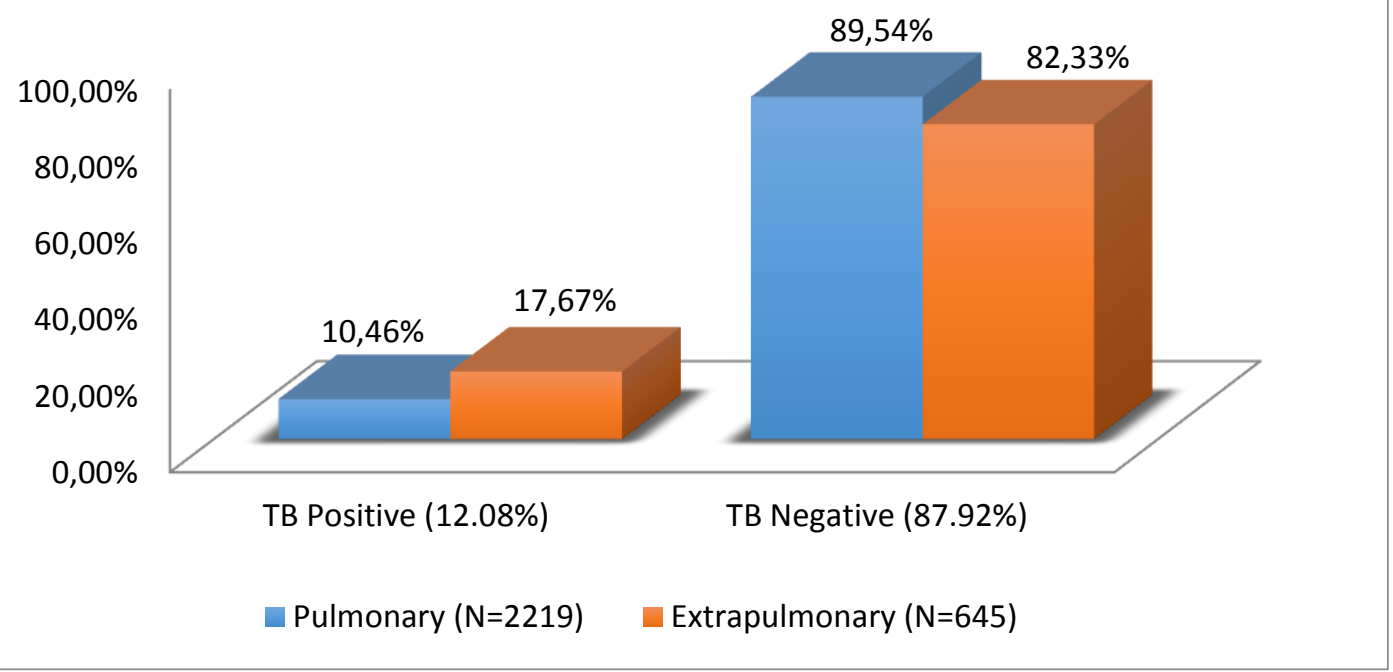

Figure 1 : Broad distribution of CBNAAT positivity $(n=2864)$.

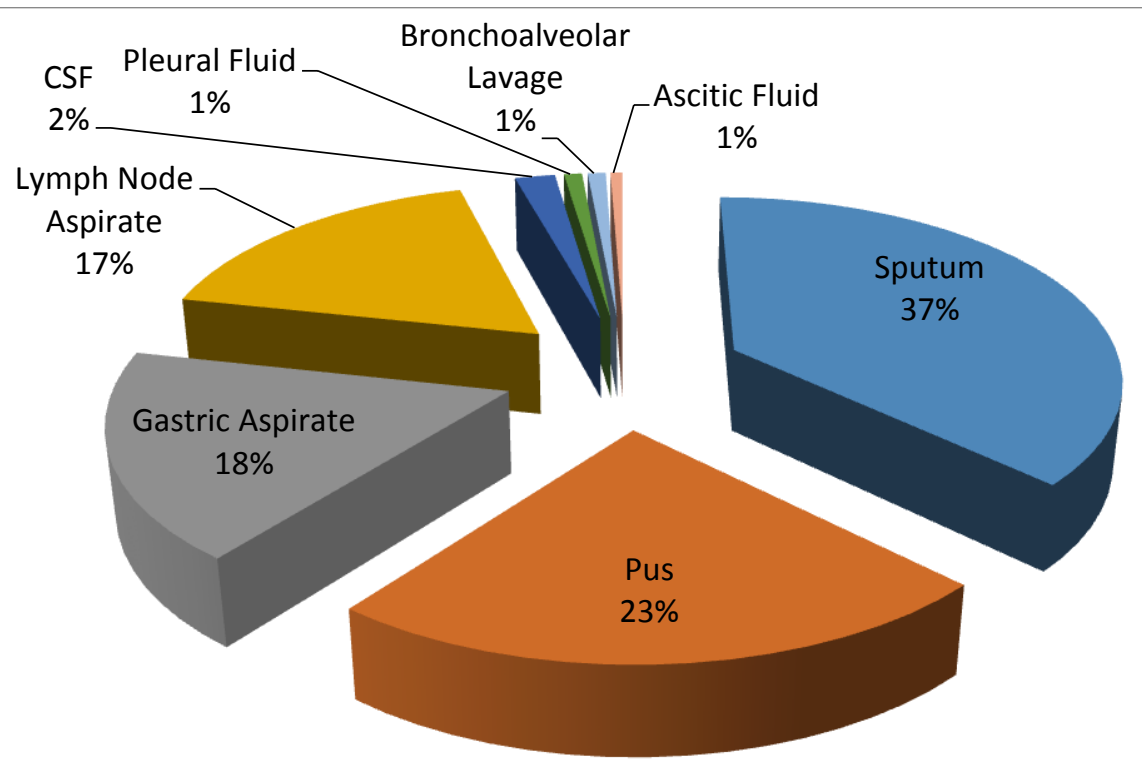

Figure 2. Distribution of CBNAAT positivity contribution according to samples $(n=346)$.

Table 3. Distribution of susceptibility of Rifampicin according to Positive CBNAAT samples ( $n=346)$.

\begin{tabular}{|l|l|l|l|l|}
\hline Variables & Source of M. tuberculosis & Rifampicin-Resistant & Rifampicin-Sensitive & Total \\
\hline Positives & Pulmonary & $13.36 \%$ & $86.64 \%$ & $100.0 \%$ \\
\hline
\end{tabular}




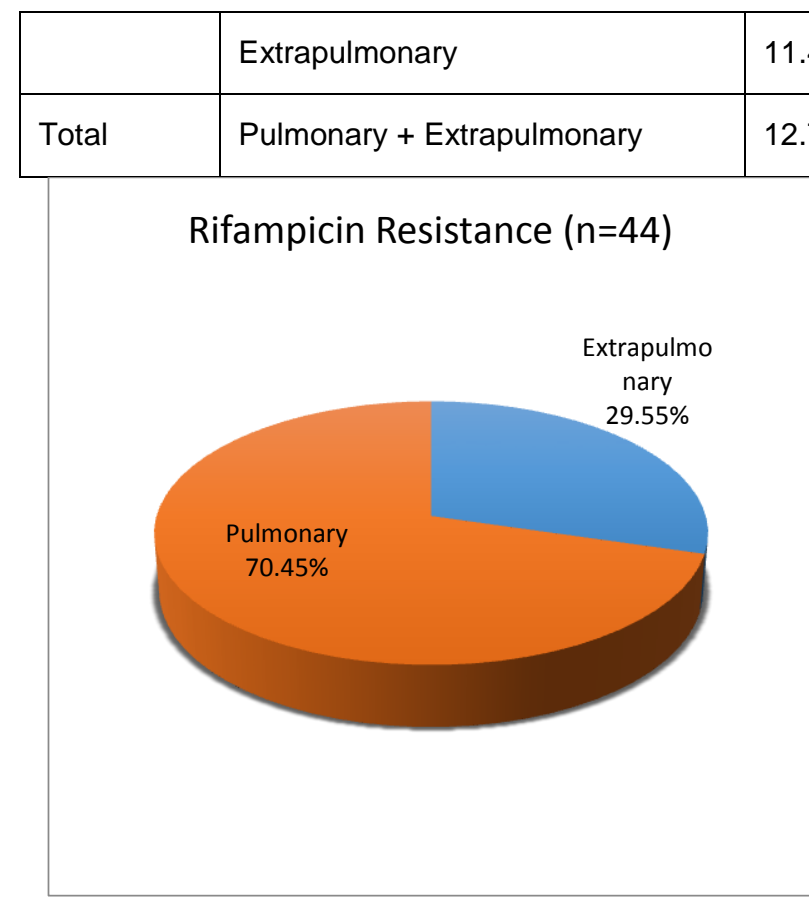

Figure 3. Distribution of TB positivity among Rifampicin resistance samples.

There is as such no proper reason for the increasing cases in this age group. A possible hypothetical reason may be the trigger induced by various variables in cognition or behavior may have led to tuberculosis. Among the extrapulmonary samples, Lymph node aspirate has shown the highest positivity which is almost similar (42.8\%) according to a study done in Bangalore, India [24].

By using Xpert MTB/RIF assay, including Rifampicin resistance, a surrogate marker for MDR strains, we observed RIF resistance which is a surrogate marker of MDR-TB in $12.7 \%$ of suspected cases. This finding is similar to the studies reported in Jaipur (11.09\%) [25] and Punjab (10\%) [26].

Xpert will divert treatment away from false smear-negative cases" to true" smear-negative TB cases, thereby increasing the accuracy of treatment and cost-effectiveness, while reducing the burdens of toxicity and cost of treatment in patients who do not in fact have TB. In our study, there were 144 (29.5\%) CBNAAT positive specimens that had shown negative results for Acid-fast bacilli (70\% Sensitivity and $96.1 \%$ Negative predictive value). The findings were similar to the study done in Maharashtra which had shown that $(8 / 25) 32 \%$ samples had been

\begin{tabular}{|l|l|l|}
\hline $1.40 \%$ & $88.60 \%$ & $100.0 \%$ \\
\hline $2.72 \%$ & $87.28 \%$ & $100.0 \%$ \\
\hline
\end{tabular}

missed for TB diagnosis by AFB smear which was positive for CBNAAT [27].

Extrapulmonary Tuberculosis is also a significant contributor to Tuberculosis and should be diagnosed the earliest along with pulmonary samples. In an earlier review done in an Indian study, EPTB (Extrapulmonary Tuberculosis) was estimated to constitute about 15 to 20 percent of all cases of tuberculosis [28]. This is quite similar to our study which showed $17.67 \%$ positivity for extrapulmonary samples.

Multidrug-resistant cases have also been observed to be caused by extrapulmonary Tuberculosis which poses difficulty in the treatment. Our study has shown Extrapulmonary MDR tuberculosis (MDR-EPTB) prevalence of $11.40 \%$ which is almost similar to the reported prevalence in studies in Brazil (14\%)[29] and $\operatorname{Nepal}(12.5 \%)[30]$. It was also observed that rifampicin-resistant pulmonary cases (13.36\%) were more as compared to extrapulmonary cases $(11.40 \%$ ) (Table 3$)$. A study done by Chakraborty et al in India found similar results with $13.7 \%$ from pulmonary and $8.6 \%$ from extra-pulmonary cases being rifampicin resistant [31].

\section{Conclusions}

The problem of MDR in extrapulmonary tuberculosis (EPTB) cannot be overlooked as being a major health problem. The key factor being the development of MDR- TB which might be delayed and incorrect diagnosis which can impose an extra load of drug administration and its side effect which ultimately might lead to extensively drug-resistant tuberculosis (XDRTB). Rapid diagnosis of drug resistance using Gene Xpert is crucial to the early treatment of drug-resistant tuberculosis and prevention of its transmission. This is a very useful assay for implementation in the TB control program as it is helpful for information regarding the mutation pattern of RIF resistance in MTB isolates which further provides an understanding of the epidemiology of the disease and identification of hot spots.

\section{ACKNOWLEDGMENTS}

We would like to gratefully acknowledge the immense help of all the staff of the Department 
of Microbiology, Lady Hardinge Medical College, New Delhi.

Declaration of Conflicting Interests: The authors declare that they have no conflict of interest.

Funding: Not applicable.

\section{REFERENCES}

1. Glaziou P, Floyd K, Raviglione MC. Global epidemiology of tuberculosis. Semin Respir Crit Care Med 2018; 39:271-85.

2. World Health Organisation. Global Tuberculosis Report, 2019.

3. Guidelines on programmatic management of drug resistant tuberculosis in India, RNTCP 2017.

4. Weiss $P$, Chen $W$, Cook VJ, et al. Treatment outcomes from community-based drug resistant tuberculosis treatment programs: a systematic review and meta-analysis. BMC Infect Dis 2014; 14: 333.

5. Central TB Division, Directorate General of Health Services, Ministry of Health with Family Welfare: Government of India. New Delhi: 2017. Revised National Tuberculosis Control Program. National Strategic Plan for Tuberculosis Elimination 2017-25.

6. Marais BJ, Gie RP, Schaff HS, et al. The natural history of childhood intra-thoracic tuberculosis; a critical review of literature from the pre-chemotherapy era. Int J Tuberc Lung Dis 2004; 8:392-402.

7. Jenkins HE, Tolman AW, Yuen CM, et al. Incidence of multidrug-resistant tuberculosis disease in children: systematic review and global estimates. Lancet 2014; 383:1572-9.

8. Dodd PJ, Gardiner E, Coghlan R, Seddon JA. Burden of childhood tuberculosis in 22 high-burden countries: a mathematical modelling study. Lancet Glob Health 2014; 2:e453-459.

9. Automated real-time nucleic acid amplification technology for rapid and simultaneous detection of tuberculosis and rifampicin resistance: Xpert MTB/RIF system for the diagnosis of pulmonary and extrapulmonary TB in adults and children: policy update. Geneva, World Health Organization, 2013

10. Rachow A, Clowes $P$, Saathoff E, et al. Increased and expedited case detection by Xpert MTB/RIF assay in childhood tuberculosis: a prospective cohort study. Clin Infect Dis 54. 2012: 1388-96.

11. Haldar S, Bose M, Chakrabarti $P$, et al. Improved laboratory diagnosis of tuberculosis-The Indian experience. Tuberculosis (Edinb) 2011; 91(5): 414426.

12. Ichhpujani RL, Agarwal SP, Chauhan LS. Diagnostic needs and status of new diagnostic tools for tuberculosis. In: Agarwal SP, Chauhan
LS.Tuberculosis control in India. Directorate General of Health Services, Ministry of Health and Family Welfare, Government of India, New Delhi, India, 2005, pp. 165-78.

13. Bianchi L, Galli L, Moriondo M, et al. Interferongamma release assay improves the diagnosis of tuberculosis in children. Pediatr Infect Dis J 2014; 28(6): 510-4.

14. Pai M, Nathavitharana R. Extrapulmonary Tuberculosis: New Diagnostics and New Policies. Indian J Chest Dis Allied Sci 2014; 56(2): 71-73.

15. World Health Organization (2017) Automated realtime nucleic acid amplification technology for rapid and simultaneous detection of tuberculosis and rifampicin resistance: Xpert MTB/RIF assay for the diagnosis of pulmonary and extrapulmonary TB in adults and children: policy update. World Health Organization, Geneva, Switzerland.

16. Lawn SD, Nicol MP. Xpert ${ }^{\circledR}$ MTB/RIF assay: Development, evaluation and implementation of a new rapid molecular diagnostic for tuberculosis and rifampicin resistance. Future Microbiol 2011; 6:1067 82.

17. Helb D, Jones M, Story E, et al. Rapid detection of mycobacterium tuberculosis and rifampicin resistance by use of on demand, near-patient technology. J Clin Microbiol 2010; 48:229-37.

18. Ajaykumar T, San S, Shiju S, Balasangameshwara VH, Kumar P. External Quality Assessment System for Sputum Smear Microscopy in India: Operational and Technical Implementation Aspects. NTI Bulletin 2005; 41(1 \& 2):18-32.

19. Xpert MTB/RIF Implementation Manual: Technical and Operational 'How-To'; Practical Considerations. Geneva: World Health Organization; 2014. Annex 2, Standard Operating Procedure (SOP) for processing extrapulmonary specimens (CSF, lymph nodes and other tissues) for Xpert MTB/RIF Assay.

20. Arega B, Menbere F, Getachew Y. Prevalence of rifampicin resistant Mycobacterium tuberculosis among presumptive tuberculosis patients in selected governmental hospitals in Addis Ababa, Ethiopia. BMC Infect Dis 2019; 19, 307

21. Nicol MP, Workman L, Isaacs W, et al. Accuracy of the Xpert MTB/RIF test for the diagnosis of pulmonary tuberculosis in children admitted to hospital in Cape Town, South Africa: a descriptive study. Lancet Infect Dis 2011;11(11): 819-824

22. Sekadde MP, Wobudeya E, Joloba ML, et al. Evaluation of the Xpert MTB/RIF test for the diagnosis of childhood pulmonary tuberculosis in Uganda: a cross-sectional diagnostic study. BMC Infect Dis 2013; 13:133.

23. Phuljhele S, Beck $P$, Sinha A, Saroj AK. Diagnostic accuracy of CBNAAT (Gene-Xpert) Vs liquid culture in clinically diagnosed presumptive childhood tuberculosis admitted in Pt. J.N.M. Medical 
College \& Dr. Bhim Rao Ambedkar Memorial Hospital, Raipur (C.G.). Int J Pediatr Res.2019; 6(09):467-473.

24. Jain SK, Ordonez A, Kinikar A, et al. Pediatric Tuberculosis in Young Children in India: A Prospective Study. Biomed Res Int. 2013; 2013:783698.

25. Verma D, Malhotra B, Goyal S, Shah T, Gupta $\mathrm{KN}$. Prevalence of MDR-TB and mutational pattern in rpoB gene of Mycobacterium tuberculosis at Eastern Rajasthan. Microcon 2014. $\left(38^{\text {th }}\right.$ National Conference of Indian Association of Medical Microbiologist) 2014; MP39:253.

26. Kaur R, Jindal N, Arora S, Kataria S. Epidemiology of Rifampicin Resistant Tuberculosis and Common Mutations in rpoB Gene of Mycobacterium tuberculosis: A Retrospective Study from Six Districts of Punjab (India) Using Xpert MTB/RIF Assay. J Lab Physicians 2016; 8(2):96-100.

27. Kasat S, Biradar M, Deshmukh A, Jadhav S, Deshmukh $H$. Effectiveness of CBNAAT in the diagnosis of extrapulmonary tuberculosis. Int $\mathrm{J}$ Res Med Sci2018; 6:3925-3928.

28. Sharma SK, Mohan A. Extrapulmonary tuberculosis. Indian J Med Res 2004; 120:316-53.

29. WHO (2012) Tuberculosis countries profiles. World Health Organization.

30. Gurung R, Bhattacharya SK, Pradhan B, Gurung $S$, Singh Y. Phenotypic characterisation and drug sensitivity testing of mycobacteria isolated from extrapulmonary tuberculosis. Kathmandu Univ Med J 2010; 8(29):57-61.

31. Chakraborty S, Chakraborty A, Talukder T, Mukherjee M, Chatterjee T. Prevalence of Mycobacterium tuberculosis Strains Isolated from Both Pulmonary and Extra Pulmonary Samples and Their Resistance to Rifampicin: A Study from Kolkata and Surrounding Suburbs. J Tuberculosis Res 2016; $4: 61-71$ 